\title{
Role of Surface Plasmons in the Decay of Image-Potential States on Silver Surfaces
}

\author{
A. García-Lekue, ${ }^{1}$ J. M. Pitarke, ${ }^{1,2}$ E. V. Chulkov, ${ }^{2,3}$ A. Liebsch, ${ }^{4}$ and P. M. Echenique ${ }^{2,3}$ \\ ${ }^{1}$ Materia Kondentsatuaren Fisika Saila, Zientzi Fakultatea, Euskal Herriko Unibertsitatea, \\ 644 Posta kutxatila, E-48080 Bilbo, Basque Country, Spain \\ ${ }^{2}$ Donostia International Physics Center (DIPC) and Centro Mixto CSIC-UPV/EHU, \\ Manuel de Lardizabal Pasealekua, E-20018 Donostia, Basque Country, Spain \\ ${ }^{3}$ Materialen Fisika Saila, Kimika Fakultatea, Euskal Herriko Unibertsitatea, \\ 1072 Posta kutxatila, E-20080 Donostia, Basque Country, Spain \\ ${ }^{4}$ Institut für Festkörperforschung, Forschungszentrum Jülich, 52425 Jülich, Germany
}

(Received 12 March 2002; published 8 August 2002)

\begin{abstract}
The combined effect of single-particle and collective surface excitations in the decay of image-potential states on Ag surfaces is investigated, and the origin of the long-standing discrepancy between experimental measurements and previous theoretical predictions for the lifetime of these states is elucidated. Although surface-plasmon excitation had been expected to reduce the image-state lifetime, we demonstrate that the subtle combination of the spatial variation of $s$ - $d$ polarization in $\mathrm{Ag}$ and the characteristic nonlocality of many-electron interactions near the surface yields surprisingly long image-state lifetimes, in agreement with experiment.
\end{abstract}

DOI: 10.1103/PhysRevLett.89.096401

The understanding of charge localization and electron dynamics at solid surfaces is of crucial importance in a large variety of physical and chemical phenomena, such as energy transfer, electronically induced adsorbate reactions, catalytic processes, epitaxy, and the development of new materials [1-4]. Accordingly, over the past two decades there has been a large effort to investigate the characteristic energies and linewidths of short-lived electronic excitations at metal surfaces [5-8].

A special class of electronic excitations at metal surfaces are the so-called image-potential states, which are localized on the vacuum side of the crystal but can move almost freely in a plane parallel to the surface $[9,10]$. These surface states occur when an electron is trapped between its own image potential on the vacuum side and a projected band gap of available bulk electronic states on the crystal side of the surface. Because of their well-defined physical properties, image states are useful to investigate the coupling of excited surface electronic states with the substrate in the time domain $[11,12]$.

Recently, femtosecond ultraviolet-pump and infraredprobe techniques were combined with two-photon photoemission (2PPE) to provide accurate measurements of the relaxation dynamics of image-potential states on the (100) surfaces of $\mathrm{Cu}$ and $\mathrm{Ag}[13,14]$; the lifetime of the $n=1$ image state on these surfaces was found to be $40 \pm 6$ and $55 \pm 5 \mathrm{fs}$, respectively, corresponding to linewidths of approximately 16.5 and $12 \mathrm{meV}$. Recent theoretical work based on many-body calculations of the electron selfenergy, which revealed the main factors that determine the coupling of image states to the substrate, predicted image-state lifetimes that are, in the case of $\mathrm{Cu}$ [15-17] and other metal surfaces [18-20], in good agreement with experiment, but failed to explain the measured relaxation of image-state electrons in $\mathrm{Ag}$ [20].
PACS numbers: 71.45.Gm, 73.20.At, 73.50.Gr, 78.47.+p

In this Letter, we investigate the decay of the $\operatorname{Ag}(100)$ $n=1$ image state by treating Auger-like single-particle and collective surface excitations on the same footing. A consistent treatment of these decay channels is particularly important because of the near-degeneracy of the imagestate energy with the $\operatorname{Ag}(100)$ surface plasmon. The surprising and novel result of our work is that, although the imaginary part of the image-state self-energy is enhanced due to the presence of the plasmon decay channel, interferences resulting from the nonlocality of the self-energy lead to a smaller overall image-state broadening, in agreement with experiment.

While measured image-state lifetimes are found to be systematically longer in $\mathrm{Ag}$ than in $\mathrm{Cu}$ surfaces [14], previous theoretical estimates seemed to indicate that image-state lifetimes should be shorter in the case of Ag. Although image states on $\mathrm{Ag}$ surfaces have energies that are slightly closer to the Fermi level than in the case of $\mathrm{Cu}$, both a smaller density of $s p$ valence electrons and a smaller polarizability of $d$ bound electrons in Ag are expected to yield weaker screening and, therefore, shorter image-state lifetimes in this material, as occurs in the case of bulk excitations [21,22]. Furthermore, image-state lifetimes in $\mathrm{Ag}$ were expected to be further reduced due to the opening of the surface-plasmon excitation channel [23]. As we argue below, this anticipated trend is reversed once the crucial nonlocality of the self-energy in the surface region is taken into account.

A first-principles description of electron dynamics in the bulk of noble metals shows that deviations from electron dynamics in a gas of $s p$ electrons mainly originate in the participation of $d$ electrons in the screening of electronelectron interactions [24,25]; hence, we account for the presence of occupied $d$ bands in Ag via a polarizable medium giving rise to additional screening, as suggested 
in Ref. [26], and compute the lifetime from the quasiparticle self-energy.

The inelastic lifetime of image-state electrons at a solid surface is known to be determined by electron-electron many-body interactions. On the energy-shell (i.e., neglecting the quasiparticle energy renormalization) and assuming translational invariance in the plane of the surface, which is taken to be normal to the $z$ axis, the decay rate (or lifetime broadening) of the excited state $\phi(z) e^{i \mathbf{k}_{\|} \cdot \mathbf{r}_{\|}}$ with parallel momentum $\mathbf{k}_{\|}$and energy $E=\varepsilon+k_{\|}^{2} /(2 m)$ (unless stated otherwise, we use atomic units; i.e., $e^{2}=$ $\left.\hbar=m_{e}=1\right)$ is given by [6]

$$
\tau_{\mathbf{k}_{\|}, \varepsilon}^{-1}=-2 \int d z \int d z^{\prime} \phi^{*}(z) \operatorname{Im} \Sigma_{\mathbf{k}_{\|, \varepsilon}}\left(z, z^{\prime}\right) \phi\left(z^{\prime}\right),
$$

where $\Sigma_{\mathbf{k}_{\|,},}\left(z, z^{\prime}\right)$ is the two-dimensional Fourier transform of the quasiparticle self-energy, and $m$ represents the effective mass accounting for the potential variation in the plane parallel to the surface.

In the $G W$ approximation [27], one considers only the first-order term in a series expansion of the self-energy $\Sigma$ in terms of the screened Coulomb interaction $W$. If one further replaces the Green function $G$ by that of noninteracting electrons, one finds

$$
\operatorname{Im} \Sigma_{\mathbf{k}_{\|,},}\left(z, z^{\prime}\right)=\sum_{\mathbf{q}_{\|}, \varepsilon_{f}} \phi_{f}^{*}\left(z^{\prime}\right) \operatorname{Im} W_{\mathbf{q}_{\|}, \omega}\left(z, z^{\prime}\right) \phi_{f}(z),
$$

where $\phi_{f}(z) e^{i \mathbf{k}_{\| \mid} \cdot \mathbf{r}_{\|}}$represent the available single-particle states with energy $E_{f}=\varepsilon_{f}+\left(\mathbf{k}_{\|}-\mathbf{q}_{\|}\right)^{2} /\left(2 m_{f}\right)\left(E_{F} \leq\right.$ $\left.E_{f} \leq E\right), \omega=E-E_{f}$ is the energy transfer, and $E_{F}$ is the Fermi energy. Short-range exchange-correlation effects, which are absent in the $G W$ self-energy of Eq. (2), can be included in the framework of the so-called $G W \Gamma$ approximation [28]. $G W \Gamma$ calculations for the $n=$ 1 image-state lifetime on the (100) and (111) surfaces of $\mathrm{Cu}$ were reported in Ref. [16], showing that they are very close to $G W$ calculations as long as the screened interaction $W$ is treated within the random-phase approximation (RPA).

Here we report $G W$-RPA calculations of the electron self-energy and the lifetime broadening by combining the one-dimensional model potential used in previous work $[15,16]$, which accurately accounts for the dynamics of $s p$ valence electrons [29], with the model reported in Ref. [23], in which the occupied $d$ bands are accounted for by the presence of a polarizable medium which extends up to a certain distance from the surface. Within this model, the screened interaction is of the form $W=v^{\prime} /\left(1-\chi^{0} v^{\prime}\right)$, where $v^{\prime}$ is the $d$-screened Coulomb interaction and $\chi^{0}$ is the nonlocal dynamical susceptibility of noninteracting $s p$ electrons. We emphasize that this approach provides a coherent treatment of single-particle and collective surface excitations.

The single-particle wave functions entering Eqs. (1) and (2), and those entering the expression for $\chi^{0}$, are obtained by solving the one-dimensional Schrödinger equation modeled in Ref. [29]. To derive $v^{\prime}$, we assume that the $s p$ valence electrons are embedded in a polarizable background characterized by a $z$-dependent local dielectric function $\epsilon_{d}(z, \omega)=\epsilon_{d}(\omega)$ for $z \leq z_{d}$ and $\epsilon_{d}(z, \omega)=1$ for $z>z_{d}$ and find [23]

$$
\begin{aligned}
\left.\boldsymbol{v}_{\mathbf{q}_{\|}, \omega}^{\prime}\left(z, z^{\prime}\right)=\frac{2 \pi}{q_{\|} \boldsymbol{\epsilon}_{d}\left(z^{\prime}, \omega\right)} \stackrel{\left[e^{-q_{\|}\left|z-z^{\prime}\right|}+\operatorname{sgn}\left(z_{d}-z^{\prime}\right)\right.}{ } \times \sigma_{d}(\omega) e^{-q_{\|}\left|z-z_{d}\right|} e^{-q_{\|}\left|z_{d}-z^{\prime}\right|}\right],
\end{aligned}
$$

where $\sigma_{d}=\left[\epsilon_{d}(\omega)-1\right] /\left[\epsilon_{d}(\omega)+1\right] . \epsilon_{d}(\omega)$ is related to the long-wavelength limit of the bulk dielectric function via $\epsilon(\omega)=\epsilon_{s p}(\omega)+\epsilon_{d}(\omega)-1$ (see, e.g., Ref. [30]), where $\epsilon_{s p}(\omega)$ corresponds to the long-wavelength limit of the bulk dielectric function of $s p$ valence electrons. The coordinate $z_{d}$ represents the position of the boundary up to which the polarizable medium is assumed to extend.

For comparison, we note that in a spatially uniform system the polarizable medium extends to infinity $\left(z_{d} \rightarrow\right.$ $\infty)$ and the $d$-screened Coulomb interaction is simply $v^{\prime}=$ $v / \epsilon_{d}(\omega), v$ being the bare Coulomb interaction. The exact high-density limit of Eq. (1) is then found to be, for electron energies near the Fermi level $\left(E-E_{F} \ll E_{F}\right)$, $\tau^{-1}=\tau_{\mathrm{QF}}^{-1} / \sqrt{\epsilon_{d}(\omega \rightarrow 0)}$, as suggested by Quinn [31], where $\tau_{\mathrm{QF}}$ represents the formula first derived by Quinn and Ferrel for a free-electron gas [32].

The $n=1$ image-state on $\operatorname{Ag}(100)$ is located close to the center of the projected band gap, it has a binding energy of $0.53 \mathrm{eV}$, and $\varepsilon-E_{F}=3.9 \mathrm{eV}$. Its probability-density has a maximum at $3.8 \AA$ outside the crystal edge $(z=0)$, which we choose to be located half a lattice spacing beyond the last atomic layer, and the penetration into the bulk crystal $(z<0)$ is found to be $5 \%$, as in the case of $\mathrm{Cu}(100)$. The effective mass of the $n=1$ image state on $\mathrm{Ag}(100)$ is known to be very close to the free-electron mass $(m \sim 1)$, and for all single-particle energies entering $\chi^{0}$ and $\operatorname{Im}[-\Sigma]$, we choose the effective mass $m_{f}$ to increase from our computed value of 0.45 at the bottom of the gap to the free-electron mass at the bottom of the valence band. The local dielectric function $\epsilon_{d}(\omega)$ is taken from bulk optical data [33]. The boundary of the polarizable medium is taken to be at $z_{d}=-1.5 a_{0}$ ( $a_{0}$ is the Bohr radius) inside the crystal, which was previously found to best reproduce the anomalous dispersion of Ag surface plasmons [26].

The lifetime broadening of the $n=1$ image state at the $\bar{\Gamma}$ point on $\operatorname{Ag}(100)$ originates in processes in which the image-state electron with energy $E=E_{F}+3.9 \mathrm{eV}$ decays into an empty state with energy $E_{f}$ above the Fermi level $\left(E_{F} \leq E_{f} \leq E\right)$. These processes can be realized by transferring energy and momentum to an excitation of the medium, thereby creating either a single-particle or a collective surface excitation with energy $\omega=E-E_{f}$ $(0 \leq \omega \leq 3.9 \mathrm{eV})$. Without $d$-electron screening, the surface-plasmon energy is too large $\left(\omega_{s p}=6.5 \mathrm{eV}\right)$ and 
only single-particle decay takes place; the presence of $d$ electrons reduces $\omega_{s p}$ to $\sim 3.7 \mathrm{eV}$ so that relaxation via surface plasmons becomes feasible.

Figure 1 shows the imaginary part of the self-energy $\operatorname{Im}\left[-\Sigma_{\mathbf{k}_{\|,}}\left(z, z^{\prime}\right)\right]$ for $\mathbf{k}_{\|}=0$ and $\varepsilon-E_{F}=3.9 \mathrm{eV}$, corresponding to the $n=1$ image state at the $\bar{\Gamma}$ point of the $\operatorname{Ag}(100)$ surface, as a function of $z$ and for fixed values of $z^{\prime}$. In the top panel, $z^{\prime}$ is taken to be well inside the metal $\left(z^{\prime}=-10 a_{0}\right)$, showing that $\operatorname{Im}[-\Sigma]$ has a maximum at $z=z^{\prime}$, as in the case of a homogeneous electron gas. When $z^{\prime}$ is fixed at the crystal edge $\left(z^{\prime}=0\right)$, as shown in the middle panel of Fig. $1, \operatorname{Im}[-\Sigma]$ is still found to be maximum at $z=z^{\prime}$, but the magnitude of this maximum now being enhanced because of the reduced electronic screening near the surface. The bottom panel of Fig. 1 corresponds to $z^{\prime}$ outside the metal $\left(z^{\prime}=10 a_{0}\right)$. In this case, the main peak of $\operatorname{Im}[-\Sigma]$ lags behind and remains localized at $z \sim 0$ rather than $z=z^{\prime}$, showing a highly nonlocal behavior of the imaginary part of the self-energy in the presence of a metal surface. The largest values of $\operatorname{Im}[-\Sigma]$ are obtained for $z, z^{\prime} \approx 0$, because of the high probability of single-particle and collective electronic excitations in the surface region.

The imaginary part of the image-state self-energy in the absence of $d$ electrons is also represented in Fig. 1, as obtained with $z_{d} \rightarrow-\infty$ [or, equivalently, $\epsilon_{d}(\omega)=1$ ]. Although $d$ electrons giving rise to additional screening should reduce both $W$ and $\operatorname{Im}[-\Sigma]$, this reduction is outweighed by the opening of the surface-plasmon excita-

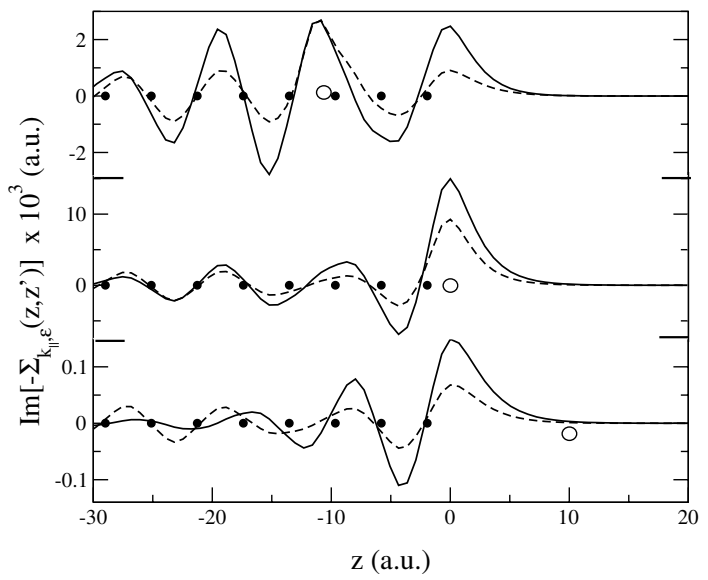

FIG. 1. Imaginary part of the $n=1$ image-state self-energy $\operatorname{Im}\left[-\Sigma_{\mathbf{k}_{\|,}, \varepsilon}\left(z, z^{\prime}\right)\right]$ versus $z$ in the vicinity of the (100) surface of $\mathrm{Ag}$. The value of $z^{\prime}$ (indicated by an open circle) is fixed at $-10 a_{0}$ (top panel), $0 a_{0}$ (middle panel), and $10 a_{0}$ (bottom panel). Notice the different scales. Solid and dashed lines represent the results obtained with $z_{d}=-1.5 a_{0}$ and with $z_{d} \rightarrow-\infty$, respectively. Full circles represent the atomic positions. The geometrical electronic edge $(z=0)$ is chosen to be located half an interlayer spacing beyond the last atomic layer. Parallel momentum and energy of the quasiparticle are taken to be $\mathbf{k}_{\|}=0$ and $\varepsilon=E_{F}+3.9 \mathrm{eV}$. tion channel which yields an overall enhancement of $\operatorname{Im}[-\Sigma]$.

Within a local picture of the self-energy and assuming, therefore, that $\operatorname{Im}[-\Sigma]$ near $z \approx z^{\prime}$ dominates the spectrum, it follows from Eq. (1) that an overall enhancement of the self-energy yields an accordingly larger expectation value of $\operatorname{Im}[-\Sigma]$, i.e., an enhanced lifetime broadening. The key point of our work is, however, that this trend is reversed, due to the characteristic nonlocality of the selfenergy near the surface. As indicated in Table I, the calculated lifetime broadening is $12 \mathrm{meV}$, while without $s d$ screening and without plasmon decay, the broadening is much larger, namely, $18 \mathrm{meV}$.

To understand this puzzling result, we note that according to Fig. 1 as long as $z^{\prime}<0$ the self-energy exhibits local character. For $z^{\prime}>0$, however, nonlocal behavior dominates. To distinguish these two spatial regions, we refer to the broadening contribution arising from $z, z^{\prime}<0$ as the "local" part and the remainder $\left(z>0\right.$ or $\left.z^{\prime}>0\right)$ as the "nonlocal" part. These contributions to the lifetime broadening are also given in Table I. We point out that nonlocal effects give rise to interference contributions to the lifetime broadening, which are determined by the specific shape of the image-state wave function in the surface region where $\operatorname{Im}[-\Sigma]$ is maximum.

This can be seen more clearly in Fig. 2 which shows the imaginary part of our calculated $n=1$ image-state selfenergy on $\operatorname{Ag}(100)$ (see also the bottom panel of Fig. 1), together with the $n=1$ image-state wave function. Since this function has a node at $z \sim 1 a_{0}$, the major interference contributions to the lifetime broadening coming from $z^{\prime}>$ 0 and $z \neq z^{\prime}$ must be negative. Furthermore, as interference dominates the image-state relaxation when $z>0$ or $z^{\prime}>$ 0 , the lifetime broadening is actually smaller than one would have expected from the penetration of the image state. According to the results given in Table I, the role that $d$ electrons play enhancing the magnitude of the imagestate self-energy is more pronounced on the vacuum side of the surface (where negative interference dominates) than in the bulk. The net effect of decay via surface plasmons and nonlocality of the self-energy is therefore a considerably reduced lifetime broadening, in excellent agreement with the time-resolved 2PPE measurement reported in Ref. [14].

TABLE I. Calculated lifetime broadening $\hbar \tau^{-1}$ in meV of the $n=1$ image state on $\operatorname{Ag}(100)$ with either $\epsilon_{d}(\omega)=1$ (or, equivalently, $\left.z_{d} \rightarrow-\infty\right)$ or $\epsilon_{d}(\omega)$ from Ref. [33] and $z_{d}=-1.5 a_{0}$. Contributions to the linewidth from excitations where $0 \leq \omega \leq$ $3.5 \mathrm{eV}$ are displayed in parentheses. The experimental linewidth is taken from time-resolved 2PPE experiments [14]. The lifetime in $\mathrm{fs}\left(1 \mathrm{fs}=10^{-15} \mathrm{~s}\right)$ is obtained by noting that $\hbar=658 \mathrm{meV} \mathrm{fs}$.

\begin{tabular}{ccccc}
\hline \hline$z_{d}$ & Local part & Nonlocal part & Total & Experiment \\
\hline$-\infty$ & $34(25)$ & $-16(-10)$ & $18(15)$ & \\
-1.5 & $59(25)$ & $-47(-15)$ & $12(10)$ & 12 \\
\hline \hline
\end{tabular}




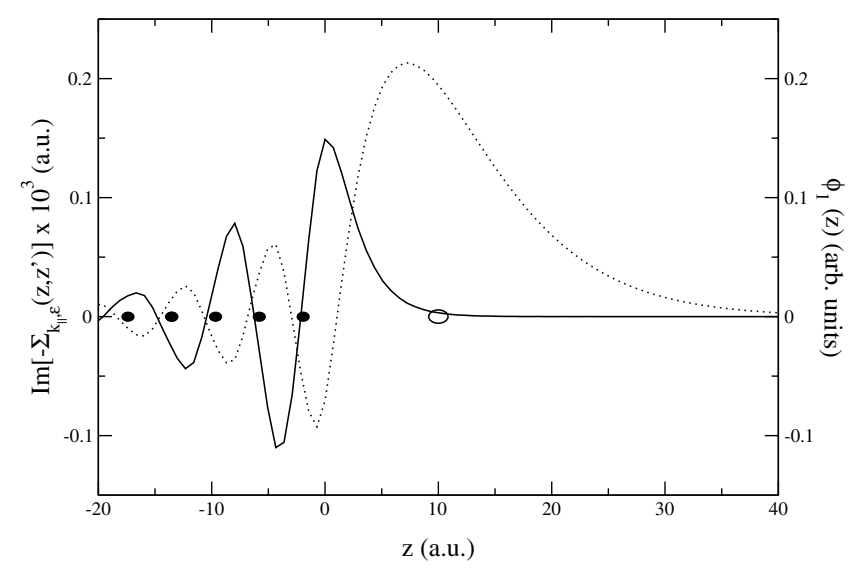

FIG. 2. Imaginary part of the $n=1$ image-state self-energy $\operatorname{Im}\left[-\Sigma_{\mathbf{k}_{\|, \varepsilon}}\left(z, z^{\prime}\right)\right]$ versus $z$ in the vicinity of the (100) surface of $\mathrm{Ag}$, for $z^{\prime}=10 a_{0}$ and $z_{d}=-1.5 a_{0}$. The dotted line represents the $n=1$ image-state wave function. As in Fig. 1, full circles represent the atomic positions and the value of $z^{\prime}$ is indicated by an open circle. $\mathbf{k}_{\|}=0$ and $\varepsilon=E_{F}+3.9 \mathrm{eV}$.

To investigate in more detail the impact of surfaceplasmon excitation on the image-state lifetime broadening, we also give in Table I the calculated linewidths obtained only from low-energy excitations $(0 \leq \omega \leq 3.5 \mathrm{eV})$ where the surface-plasmon relaxation channel is excluded (numbers in parentheses). In this case, the impact of $d$ electrons on the lifetime broadening is much smaller. Thus, the opening of the surface-plasmon relaxation channel, which occurs only when both $d$ electrons and all contributions with $0 \leq \omega \leq 3.9 \mathrm{eV}$ are included, yields a considerable enhancement of both the local and the nonlocal part of the linewidth. As the large enhancement of the negative nonlocal linewidth outweighs the increase of the local contribution, the overall influence of the occupied $d$ bands results in a reduced lifetime broadening.

In conclusion, we have investigated the influence of single-particle and collective surface excitations on the decay of image-potential states on silver surfaces, by combining an accurate description of the dynamics of $s p$ valence electrons with a physically motivated model in which the occupied $d$ bands are accounted for by the presence of a polarizable medium. Our results demonstrate that, despite the enhancement of the image-state selfenergy due to decay via surface plasmons, the highly nonlocal character of this self-energy in the surface region ultimately leads to a surprisingly small lifetime broadening, in agreement with the experimental data.
We acknowledge partial support by the University of the Basque Country, the Basque Unibertsitate eta Ikerketa Saila, the Spanish Ministerio de Educación y Cultura, and the Max Planck Research Award Funds.

[1] N.-H. Ge et al., Science 279, 202 (1998).

[2] M. Wolf and G. Ertl, Science 288, 1352 (2000).

[3] H. Petek et al., Science 288, 1402 (2000).

[4] H. Nienhaus, Surf. Sci. Rep. 45, 1 (2002).

[5] Th. Fauster and W. Steinmann, in Photonic Probes of Surfaces, edited by P. Halevi (Elsevier, Amsterdam, 1995).

[6] P. M. Echenique et al., Chem. Phys. 251, 1 (2000).

[7] X. J. Shen et al., Chem. Phys. Lett. 351, 1 (2002).

[8] M. Roth et al., Phys. Rev. Lett. 88, 096802 (2002).

[9] P. M. Echenique and J. B. Pendry, J. Phys. C 11, 2065 (1978); Prog. Surf. Sci. 32, 111 (1990).

[10] N. V. Smith, Rep. Prog. Phys. 51, 1227 (1988).

[11] E. W. Plummer, Science 277, 1447 (1997).

[12] S. Ogawa et al., Phys. Rev. Lett. 82, 1931 (1999).

[13] U. Höfer et al., Science 277, 1480 (1997).

[14] L. Shumay et al., Phys. Rev. B 58, 13974 (1998).

[15] E. V. Chulkov et al., Phys. Rev. Lett. 80, 4947 (1998); J. Osma et al., Phys. Rev. B 59, 10591 (1999).

[16] I. Sarria et al., Phys. Rev. B 60, 11795 (1999).

[17] W. Berthold et al., Phys. Rev. Lett. 88, 056805 (2002).

[18] A. Schäfer et al., Phys. Rev. B 61, 13159 (2000).

[19] S. Link et al., Phys. Rev. B 63, 115420 (2001).

[20] P. M. Echenique et al., Appl. Phys. A 71, 503 (2000).

[21] R. Keyling et al., Phys. Rev. B 61, 1670 (2000).

[22] V. P. Zhukov et al., Phys. Rev. B 64, 195122 (2001).

[23] C. López-Bastidas et al., Phys. Rev. B 65, 035417 (2001).

[24] I. Campillo et al., Phys. Rev. Lett. 83, 2230 (1999); I. Campillo et al., Phys. Rev. B 61, 13484 (2000).

[25] I. Campillo et al., Phys. Rev. B 62, 1500 (2000).

[26] A. Liebsch, Phys. Rev. Lett. 71, 145 (1993); Phys. Rev. B 48, 11317 (1993). See also Electronic Excitations at Metal Surfaces (Plenum, New York, 1997), p. 94.

[27] L. Hedin and S. Lundqvist, Solid State Phys. 23, 1 (1969).

[28] G. D. Mahan and B. Sernelius, Phys. Rev. Lett. 62, 2718 (1989).

[29] E. V. Chulkov et al., Surf. Sci. 391, L1217 (1997).

[30] H. Ehrenreich and H. R. Philipp, Phys. Rev. 128, 1622 (1962).

[31] J. J. Quinn, Appl. Phys. Lett. 2, 167 (1963).

[32] J. J. Quinn and R. A. Ferrell, Phys. Rev. 112, 812 (1958).

[33] P. B. Johnson and R. W. Christy, Phys. Rev. B 6, 4370 (1972). 